\title{
Processo Patológico do Transtorno de Ansiedade Segundo a Literatura Digital Disponível em Português - Revisão Integrativa
}

\author{
Pathological Process of Anxiety Disorder According to the Digital Literature Available in \\ Portuguese - Integrative Review
}

\section{Procedimiento Patológico del Trastorno de Ansiedad Según la Literatura Digital \\ en Portugués - Revisión Integradora}

\author{
Lívia Ivo D'ávila \\ Fernanda Cardoso Rocha \\ Bruna Roberta Meira Rios \\ Sabrina Gonçalves Silva Pereira \\ Álvaro Parrela Piris \\ Faculdade de Saúde Ibituruna
}

\begin{abstract}
Resumo
Com o objetivo de discutir as possibilidades estratégicas quanto aos processos patológicos que envolvem o transtorno de ansiedade, para embasar os profissionais quanto à ansiedade e aos seus tipos, este estudo configura-se como uma pesquisa de caráter integrativo sistematizado, com uma busca on-line das produções científicas na base de dados Biblioteca Virtual de Saúde, utilizando os descritores "Transtorno de ansiedade", "Ansiedade" e "Tratamento da ansiedade", veiculados em periódicos de estratos de $\mathrm{A} 1, \mathrm{~A} 2, \mathrm{~B} 1, \mathrm{~B} 2, \mathrm{~B} 3$ e B4, disponíveis para download na íntegra, escritos na língua portuguesa. Os achados identificam que as possibilidades estratégicas perante esse transtorno se dão por duas categorias, a Terapia Cognitivo-Comportamental e o Tratamento Humanizado e Apoio Familiar, sendo este último mais eficaz quando associado ao uso de outros tratamentos para a ansiedade. Ressalta-se a importância de estudos e capacitação para atuar efetivamente na prevenção e no tratamento dessa patologia, além de mais estudos sobre a temática.
\end{abstract}

Palavras-chave: ansiedade, saúde mental, psicopatologia, tratamento

\begin{abstract}
With the objective of discussing the strategic possibilities regarding the pathological processes that involve anxiety disorder, to support the professionals about anxiety and its types, this study is configured as a systematized integrative research, with an online search of scientific productions in the Virtual Health Library database, using the descriptors "Anxiety disorder", "Anxiety" and "Anxiety treatment", published in journals of strata of A1, A2, B1, B2, B3, and B4, available for download, written in the Portuguese language. The findings identify that the strategic possibilities for this disorder are given by two categories, Cognitive Behavioral Therapy and Humanized Treatment and Family Support, the latter being more effective when associated with the use of other treatments for anxiety. The importance of studies and training to effectively act in the prevention and treatment of this pathology is emphasized, as well as more studies on the subject.
\end{abstract}

Keywords: anxiety, mental health, psychopathology, treatment

\section{Resumen}

Con el objetivo de discutir las posibilidades estratégicas en cuanto a los procesos patológicos que involucran el trastorno de ansiedad, para basar a los profesionales en cuanto a la ansiedad y sus tipos, este estudio se configura como una investigación de carácter integrativo sistematizado, con una búsqueda online de las producciones científicas en la base de datos Biblioteca Virtual de Salud, utilizando los descriptores "Trastorno de ansiedad", "Ansiedad" y "Tratamiento de la ansiedad", publicados en periódicos de estratos de A1, A2, B1, B2, B3 y B4, disponibles para descargar en la base de datos en la íntegra, escritos en portugués. Los hallazgos identifican que las posibilidades estratégicas ante este trastorno se dan por dos categorías, la Terapia Cognitiva Comportamental y el Tratamiento Humanizado y Apoyo Familiar, siendo ese último más eficaz cuándo asociado al uso de otros tratamientos para la ansiedad. Se resalta la importancia de estudios y capacitación para actuar

\footnotetext{
${ }^{1}$ Endereço de contato: Av. Profa. Aida Mainartina Paraíso, 99, Ibituruna, Montes Claros, MG, CEP 39408-007. E-mail: liviaidavila1@gmail.com
} 
efectivamente en la prevención y tratamiento de esa patología, además de más estudios sobre la temática.

Palabras clave: ansiedad, salud mental, la psicopatología, tratamiento

\section{Introdução}

São percebidas ao longo do tempo diversas concepções a respeito da ansiedade, as quais se vinculam a uma visão que a analisa de modo completo em relação ao esquema de saúdedoença, como os estudos egípcios e gregos acerca das doenças no mundo, uns dos pioneiros nos estudos referentes à medicina mundial. Os egípcios acreditavam que a doença era uma possessão demoníaca ou até mesmo um encantamento, intrusão de espíritos, sortilégio, e rituais terapêuticos eram realizados com o intuito de fazer com que a doença saísse do indivíduo que o apoderou. Com isso, foi uma das primeiras concepções no mundo que trazia afirmativas e estudos relevantes à época quanto aos agravos e doenças sofridos, também fazendo referências a outras afecções de saúde, como a parasitária e a infecciosa (Canguilhem, 2009).

Por meio das concepções da medicina grega, são estudadas também as diferenças entre a saúde e a doença, estabelecendo conceitos que, por volta do século IV a.C., passaram a fazer parte da dinâmica ontológica das doenças. Desta forma, acreditavam que a doença era um desequilíbrio da natureza do indivíduo, que se estabelecia por meio de tais percepções, nas quais determinadas substâncias produzidas pelo organismo provocavam o desequilíbrio no corpo do indivíduo, sendo elas conhecidas como os quatro humores corporais, do sangue, da bile negra, da bile amarela e da linfa, respectivamente relacionados ao coração, baço, fígado e fleura ou cérebro. Assim, acreditava-se que a natureza podia interferir em tais aspectos e provocar as doenças (Canguilhem, 2009).

Posto isso, diversas foram as concepções que englobavam a doença ao longo do tempo, que provocaram grandes transformações no mundo. Assim, em relação aos estados ansiosos, são analisadas algumas informações que antecedem ao nascimento de Cristo (Nardi, 2006). Desperta, assim, com o decorrer dos tempos, o interesse da medicina moderna, transformando-se em um quadro patológico específico.

Segundo Nardi (2006), já eram encontradas provas sobre a existência da ansiedade no século VIII a.C., na Ilíada, obra do poeta consagrado Homero, em que ele descreve sentimentos de medo, perturbações, intrusão de Deus, entre outros descritos em sua obra.

Com isso, são conhecidas diversas representações e alusões em toda a história da sociedade. Na atualidade, as obras de diversos autores refletem os estudos sobre o transtorno de ansiedade generalizada (TAG), nos quais afirmam novas perspectivas de conhecimento que tiram a ideia de perturbações dos deuses ou de que essas pessoas têm alguma representação negativa de maldade e incorporações demoníacas, dando espaço para o estudo do ser como um todo, cheio de singularidades (Nardi, 2006).

Para Castillo, Recondo, Asbahr e Manfro (2000), em relação ao transtorno de ansiedade, este se caracteriza como um sentimento que muitas vezes se torna algo vago que a pessoa sente, de modo desagradável, sendo relacionado com o medo, apreensão ou até desconfiança sentida advinda da tensão, desconforto e, principalmente, antecipação do sentimento de perigo contínuo de algo estranho ou desconhecido, em que há sensações de preocupação 
constantes, de forma que é gerada uma insegurança contínua e desconfiança em tudo e todos.

Quando o transtorno de ansiedade é identificado nas crianças, de forma a influenciar diretamente em seu desenvolvimento emocional, psicológico, em que seus pensamentos se determinam a partir do medo, muitas vezes de forma irracional, pois isso ainda se enquadra nas ações do desenvolvimento de diversas funções e perspectivas em relação a sua personalidade, bem como a constantes preocupações, podendo ser normais ou patologias desenvolvidas, faz-se com que haja desconfianças constantes sobre sua saúde, segurança, alimentação, vida social, escola e sua personalidade (Andrade, Viana, \& Silveira, 2006).

Com isso, segundo as concepções de Dalgalarrondo (2000), a psicopatologia pode ser definida como o conjunto de fundamentos alusivo ao adoecimento mental do ser humano. 0 autor afirma ainda ser um conhecimento sistemático, elucidativo e desmistificante, que não inclui princípios de valor e recusa dogmas ou verdades a priori.

Assim, por meio de um levantamento feito em 2016 sobre depressão, publicado pela Organização Mundial da Saúde, compreende-se que 264 milhões de pessoas no mundo sofrem com o transtorno de ansiedade, em diversos tipos e graus. Aponta-se que o Brasil tem mais de $9 \%$ da população com algum tipo de transtorno de ansiedade, ou seja, quase três vezes mais que a média mundial. São mais de 18 milhões de pessoas nessa situação (Organização Mundial da Saúde [OMS], 2016).

Verificando os índices de acometimento do transtorno de ansiedade em relação ao sexo, foi constatado, segundo Kinrys e Wygant (2005), que as mulheres são as mais suscetíveis a terem o transtorno ao longo da vida adulta.

O transtorno de ansiedade é um dos temas da psiquiatria mais subdiagnosticados na atualidade, pois são raras as vezes em que um indivíduo procura por um atendimento completo e profissional dentro dos aspectos da saúde mental no Brasil. Com isso, são estabelecidos alguns sintomas que auxiliam na identificação do transtorno, porém esses, um tanto vagos e não claramente fonte de diagnóstico para a ansiedade, são sintomas físicos pouco aparentes e pouco definidos (Zuardi, 2017).

A utilização dos critérios diagnósticos do DMS-V auxilia numa melhor classificação da ansiedade, identificando que, com o desenvolvimento da ansiedade, há um maior envolvimento nos inúmeros eventos das atividades cotidianas. A ansiedade e a preocupação estão associadas a três ou mais sintomas, presentes na maioria dos dias, nos últimos seis meses, sendo os mais identificados a inquietação ou sensação de estar no limite, cansar-se facilmente, dificuldade de concentração; irritabilidade, tensão muscular e distúrbios do sono. Dessa forma, o transtorno não pode ser atribuído a apenas uma condição médica geral, mas também ao transtorno mental (Zuardi, 2017).

Em análises dos fatores relacionados a problemas e/ou transtornos referentes a indivíduos que sofrem com a ansiedade crônica, percebe-se que os fatores de risco mais abrangentes são em relação ao gênero, e, por meio dos estudos, indica-se que pessoas do sexo feminino são as mais acometidas pela ansiedade, em relação a mudanças de humor e estresse. Os traumas também caracterizam uma pessoa com ansiedade crônica, em relação a abusos sofridos na infância e a eventos traumáticos. As doenças e comorbidades também indicam a presença do transtorno, como o câncer, que é uma das maiores afecções e preocupações 
da população atual. Assim como a personalidade, com pessoas mais tensas, preocupadas e a presença de outros tipos de transtorno concomitantes. A genética e o abuso de substâncias podem levar ao desenvolvimento ou ao agravamento da condição da ansiedade em relação à pessoa, em que o indivíduo aumenta seus riscos e agravos e a sua prevalência (Craske, Stein, \& Hermann, 2014).

Com isso, existem diversas complicações acerca do transtorno de ansiedade, as quais influenciam nas condições de vida do indivíduo que sofre diariamente com isso, sendo caracterizadas muitas vezes pela depressão, abuso de substâncias como drogas ilícitas e álcool, problemas digestivos ou intestinais, dores de cabeça, enxaquecas, bruxismo, transtornos por uso de substância, entre outros, que podem levar a casos mais graves, como o suicídio, que cada vez mais atinge essas pessoas (Zuardi, 2017).

De acordo com as afirmações de Castillo et al. (2000), os transtornos ansiosos caracterizam-se com sintomas primários, não sendo derivados de outros tipos de casos psiquiátricos, como depressões, psicoses, transtornos do desenvolvimento, transtorno hipercinético, entre outros, porém os sintomas de transtornos ansiosos ocorrem em outros casos da psiquiatria. Os autores afirmam, ainda, que há alguns casos em que a ansiedade se explica por meio dos transtornos ansiosos, sendo eles a ansiedade do início do surto esquizofrênico e o temor da separação dos pais para uma criança com depressão maior, por exemplo, não estabelecendo um conjunto de sintomas que ocasionam um transtorno ansioso típico (Castillo et al., 2000).

Isso mostra que os sintomas do transtorno de ansiedade não se caracterizam unicamente pela ansiedade propriamente dita, mas podem ocorrer alguns sintomas que caracterizam outros tipos de transtornos, todos ao mesmo tempo, enquadrando a pessoa em diversas possibilidades diagnósticas (Castillo et al., 2000).

Diante de tantas informações referentes a essa condição, encontram-se ainda grandes desafios a serem derrubados, para que as pessoas que apresentam o TAG possam ter a oportunidade de serem compreendidas e para que complicações mais severas não ocorram, como o já citado suicídio. Assim, torna-se imprescindível a valorização do indivíduo em suas particularidades, pois não há em lugar algum alguém que seja igual ao outro na personalidade, nos sentimentos, nos aspectos pessoais e nas vivências que o levam para um caminho incerto e profundamente caótico. Dessa forma, é necessária uma avaliação mais profunda por profissionais, levando em consideração a orientação, o aconselhamento, a utilização de técnicas de relaxamento e/ou exercícios físicos, não se limitando a prescrever medicamentos (Zuardi, 2017).

Nessa perspectiva, pode-se observar que o transtorno de ansiedade engloba diversos aspectos da personalidade psíquica e emocional do indivíduo que o tem; considerando ainda suas possibilidades diagnósticas que envolvem outros diversos transtornos, torna-se essencial ao profissional da área da saúde se preparar adequadamente para a compreensão e o auxílio dessas pessoas, pois é uma tarefa que tem grandes dificuldades. A perspectiva da multidisciplinaridade se torna algo primordial, fazendo com que profissionais envolvidos na saúde mental, principalmente, tenham o compromisso de estarem devidamente preparados para a eficaz compreensão e auxílio a seus pacientes, fator essencial a um tratamento concreto e acompanhamento eficiente. Portanto, a partir dos fatos apresentados, cabe neste estudo a construção de uma revisão do tipo integrativa da literatura para embasar os 
profissionais quanto à ansiedade. Desta forma, este estudo busca responder a problemática de quais possíveis tratamentos apresentados pela literatura são mais efetivos ao TAG. Como objetivo, este estudo busca identificar formas de tratamento mais eficazes ao TAG, por meio da literatura.

\section{Método}

Com o objetivo de discutir as possibilidades estratégicas quanto aos processos patológicos que envolvem o transtorno de ansiedade, para embasar os profissionais quanto à ansiedade e aos seus tipos, este estudo configura-se como uma pesquisa de caráter integrativo sistematizado, com o estudo de achados que estão relacionados ao tema definido, com o intuito de analisar, avaliar e agregar de forma sistemática e sucinta os resultados encontrados, com base em aspectos inerentes ao transtorno de ansiedade.

De acordo com as concepções de Sampaio e Mancini (2007), trata-se de um estudo de caráter integrativo sistematizado, assim como outros tipos de estudo de revisão, com uma forma de pesquisa que utiliza as fontes de dados da literatura sobre um tema definido. A realização desse tipo de trabalho de análise e investigação disponibiliza um resumo das evidências relacionadas a uma estratégia de intervenção específica. Afirmam ainda que, por meio da utilização de métodos explícitos e sistematizados de busca, apreciação crítica e síntese da informação selecionada, aplica-se uma revisão crítica das informações coletadas.

Assim, com a formulação dos descritores de ciências da saúde (DeCs), foi realizada a busca on-line das produções científicas na base de dados Biblioteca Virtual de Saúde (BVS), utilizando os descritores "Transtorno de ansiedade", "Ansiedade" e "Tratamento da ansiedade".

Foram incluídos artigos disponíveis para download na íntegra, escritos na língua portuguesa, veiculados em periódicos de estratos de A1, A2, B1, B2, B3 e B4, preferencialmente revistas científicas com temáticas na área da psicologia e/ou da saúde, publicadas entre janeiro de 2010 e dezembro de 2017. Foram excluídos trabalhos não publicados no tempo determinado, os que não estavam disponíveis gratuitamente para download, os que não estavam na língua portuguesa brasileira e os que fugiam da temática proposta.

Visando à qualidade metodológica dos artigos, esses foram selecionados após análise minuciosa dos autores desta pesquisa, baseando nas diretrizes do Preferred Reporting Items for Systematic Reviews and Meta-Analyses (Moher, Liberati, Tezlaff, Altman, \& The PRISMA Group, 2009). 


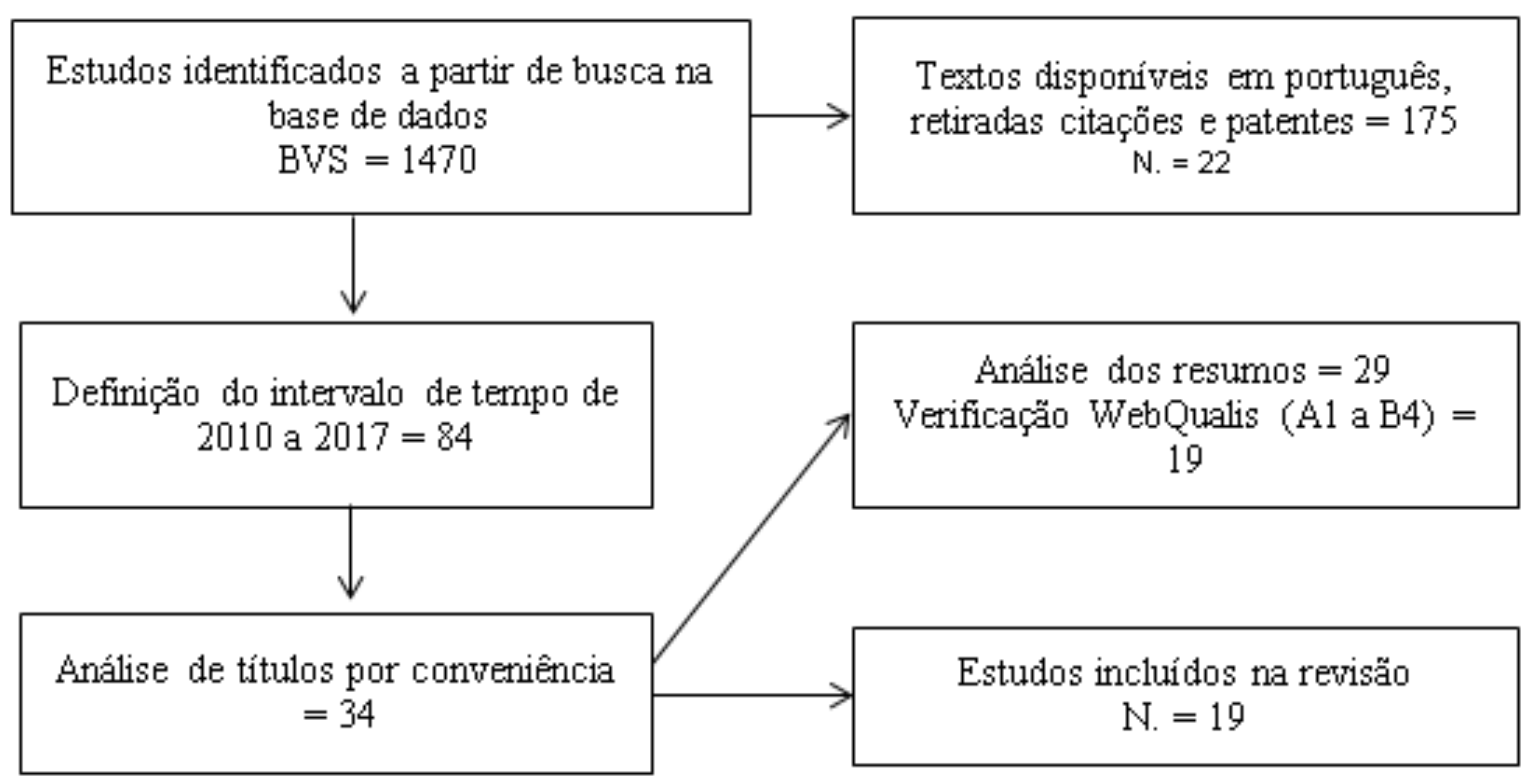

Quadro 1. Fluxograma de seleção dos estudos.

Tabela 1

Coleta de dados

\begin{tabular}{|c|c|c|c|c|}
\hline Nome do artigo & Publicação & Metodologia & Objetivo & WebQualis \\
\hline $\begin{array}{l}\text { A Terapia Cogniti- } \\
\text { vo-Comportamental } \\
\text { em Grupo no Tran- } \\
\text { storno de Ansiedade } \\
\text { Social }\end{array}$ & $\begin{array}{l}\text { Revista Estud. } \\
\text { Pesqui. Psicol. } \\
\text { v. } 15 \text { | n. } 3 \text { | p. } \\
1061-1080 \text { | } \\
2015 .\end{array}$ & $\begin{array}{c}\text { Estudo de campo com } \\
\text { cinco voluntários acima } \\
\text { de } 18 \text { anos na cidade de } \\
\text { Juiz de Fora. }\end{array}$ & $\begin{array}{c}\text { Descrever o desen- } \\
\text { volvimento de uma } \\
\text { intervenção em grupo, } \\
\text { baseada na Terapia Cog- } \\
\text { nitivo-Comportamental, } \\
\text { para pessoas que apre- } \\
\text { sentaram os sintomas } \\
\text { de TAS. }\end{array}$ & $A 2$ \\
\hline $\begin{array}{l}\text { Adesão ao Tratamento } \\
\text { Medicamentoso por } \\
\text { Pessoas com Transtorno } \\
\text { de Ansiedade }\end{array}$ & $\begin{array}{l}\text { Revista Cog- } \\
\text { itare Enfer- } \\
\text { magem | v. } 21 \\
\text { | n. } 1 \text { | p. 1-11 } \\
\text { | jan./mar. } \\
\text { 2016. }\end{array}$ & $\begin{array}{l}\text { Estudo quantitativo, } \\
\text { transversal, descriti- } \\
\text { vo, realizado com } 161 \\
\text { pessoas de um serviço } \\
\text { de saúde mental, num } \\
\text { município do estado de } \\
\text { São Paulo, entre 10 de } \\
\text { janeiro e } 31 \text { de dezem- } \\
\text { bro de } 2012 \text {. }\end{array}$ & $\begin{array}{l}\text { Verificar os fatores } \\
\text { relacionados à adesão de } \\
\text { pessoas com transtorno } \\
\text { de ansiedade quanto à } \\
\text { farmacoterapia prescrita. }\end{array}$ & B2 \\
\hline $\begin{array}{c}\text { Avaliação de Ansiedade e } \\
\text { Depressão em Pacientes } \\
\text { Oncológicos: Com- } \\
\text { paração Psicométrica }\end{array}$ & $\begin{array}{l}\text { Revista Psico - } \\
\text { USF | v. } 19 \mid \mathrm{n} . \\
2 \text { | maio/ago. } \\
\text { 2014. }\end{array}$ & $\begin{array}{c}\text { Estudo comparativo } \\
\text { descritivo. }\end{array}$ & $\begin{array}{l}\text { Comparar vantagens e } \\
\text { desvantagens psicométri- } \\
\text { cas de instrumentos } \\
\text { comumente utilizados } \\
\text { em serviços especializa- } \\
\text { dos em Oncologia. }\end{array}$ & $\mathrm{A} 2$ \\
\hline
\end{tabular}




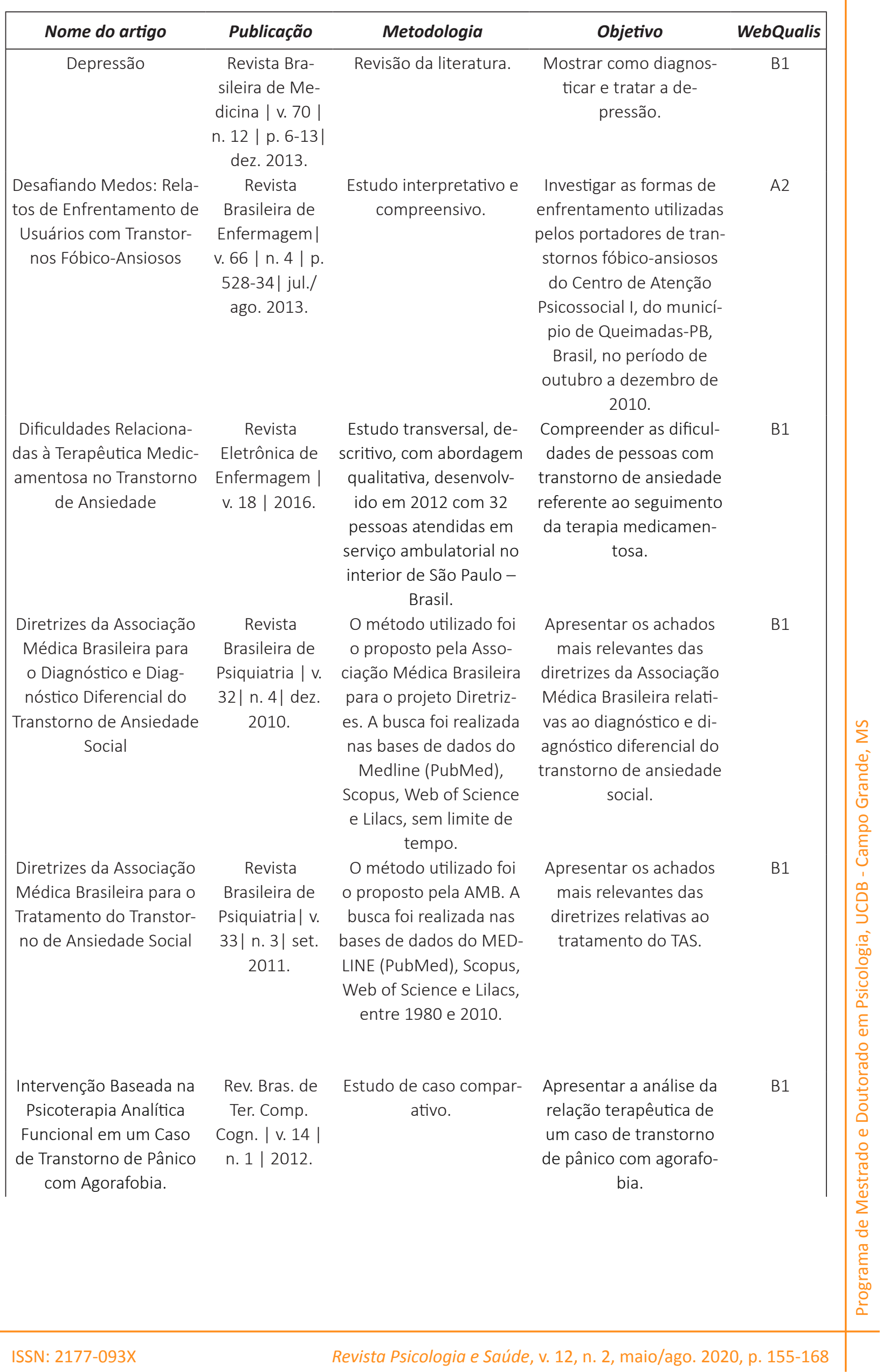




\begin{tabular}{|c|c|c|c|c|}
\hline Nome do artigo & Publicação & Metodologia & Objetivo & WebQualis \\
\hline $\begin{array}{l}\text { Periódicos da CAPES: } \\
\text { Perspectiva das Disser- } \\
\text { tações e Teses sobre } \\
\text { Transtorno de Ansiedade } \\
\text { Social/Fobia Social }\end{array}$ & $\begin{array}{c}\text { Revista } \\
\text { Interação em } \\
\text { Psicologia | } \\
\text { v. } 16 \mid \text { n. } 2 \text { | } \\
\text { p. } 283-292 \text { | } \\
2012 .\end{array}$ & $\begin{array}{l}\text { Estudo de revisão bib- } \\
\text { liográfica, descritivo e } \\
\text { comparativo. }\end{array}$ & $\begin{array}{c}\text { Fornecer um panorama } \\
\text { das produções acadêmi- } \\
\text { cas referentes a teses e } \\
\text { dissertações da CAPES, } \\
\text { defendidas entre } 1987 \text { e } \\
2009 .\end{array}$ & B2 \\
\hline $\begin{array}{l}\text { Psicologia e Profissão: } \\
\text { Neurose Profissional e } \\
\text { a Atuação do Psicólogo } \\
\text { Organizacional Frente à } \\
\text { Questão }\end{array}$ & $\begin{array}{l}\text { Revista Psico- } \\
\text { logia: Ciência } \\
\text { e Profissão | } \\
\text { v. } 30 \mid \text { n. } 2 \text { | } \\
\text { p. } 248-261 \mid \\
2010 .\end{array}$ & Revisão bibliográfica. & $\begin{array}{l}\text { Realizar um levanta- } \\
\text { mento bibliográfico } \\
\text { sobre a neurose profis- } \\
\text { sional, cujos sintomas } \\
\text { são entendidos como a } \\
\text { expressão simbólica de } \\
\text { um conflito psíquico que } \\
\text { se desenvolve a partir } \\
\text { de uma situação organ- } \\
\text { izacional ou profissional } \\
\text { determinada. }\end{array}$ & $\mathrm{A} 2$ \\
\hline $\begin{array}{c}\text { Saúde Mental, Gênero e } \\
\text { Violência Estrutural }\end{array}$ & $\begin{array}{l}\text { Revista Bioéti- } \\
\text { ca | v. } 20 \mid \mathrm{n} . \\
2 \mid \text { p. } 267-79 \mid \\
2012 .\end{array}$ & $\begin{array}{l}\text { Estudo de caso realiza- } \\
\text { do por meio de análise } \\
\text { quantitativa de pron- } \\
\text { tuários de pacientes. }\end{array}$ & $\begin{array}{l}\text { Analisar os sintomas e } \\
\text { diagnósticos encontra- } \\
\text { dos em prontuários de } \\
\text { pacientes homens e mul- } \\
\text { heres de dois grandes } \\
\text { hospitais psiquiátricos do } \\
\text { Distrito Federal. }\end{array}$ & A2 \\
\hline $\begin{array}{l}\text { Subtipo Respiratório ver- } \\
\text { sus Não Respiratório no } \\
\text { Transtorno de Pânico } \\
\text { com Agorafobia: Aval- } \\
\text { iação com Terapia Cogni- } \\
\text { tivo-Comportamental }\end{array}$ & $\begin{array}{l}\text { Revista Psico- } \\
\text { logia: Reflexão } \\
\text { e Crítica | v. } 25 \\
\text { | n. } 1 \text { | } 2012 .\end{array}$ & $\begin{array}{l}\text { Amostra randomizada } \\
\text { por sorteio com } 50 \text { pa- } \\
\text { cientes diagnosticados } \\
\text { segundo o Manual Diag- } \\
\text { nóstico e Estatístico dos } \\
\text { Transtornos Mentais. }\end{array}$ & $\begin{array}{l}\text { Verificar a resposta dos } \\
\text { pacientes com tran- } \\
\text { storno de pânico com } \\
\text { agorafobia ao modelo } \\
\text { proposto de Terapia Cog- } \\
\text { nitivo-Comportamental } \\
\text { (TCC) nos dois subtipos } \\
\text { respiratórios de transtor- } \\
\text { no de pânico. }\end{array}$ & A1 \\
\hline $\begin{array}{l}\text { Terapia Cognitivo-Com- } \\
\text { portamental em Pa- } \\
\text { cientes com Púrpura } \\
\text { Idiopática }\end{array}$ & $\begin{array}{l}\text { Rev. Bras. de } \\
\text { Ter. Comp. } \\
\text { Cogn. | v. 18 | } \\
\text { n. } 3 \mid \text { p. } 58-69 \mid \\
2016 .\end{array}$ & $\begin{array}{l}\text { Estudo baseado em } 10 \\
\text { sessões de psicoterapia } \\
\text { e a utilização de uma es- } \\
\text { cala para medir a inten- } \\
\text { sidade dos sintomas de } \\
\text { ansiedade, o inventário } \\
\text { de ansiedade Beck (BAI), } \\
\text { juntamente da coleta de } \\
\text { exames de sangue. }\end{array}$ & $\begin{array}{l}\text { Tratar, por meio da Tera- } \\
\text { pia Cognitivo-Comporta- } \\
\text { mental, o transtorno de } \\
\text { ansiedade de pacientes } \\
\text { que se encontram em } \\
\text { tratamento médico, cujo } \\
\text { diagnóstico é de púrpura } \\
\text { idiopática. }\end{array}$ & B1 \\
\hline $\begin{array}{l}\text { Toxina Botulínica como } \\
\text { Tratamento para Fobia } \\
\text { Social Generalizada com } \\
\text { Hiperidrose }\end{array}$ & $\begin{array}{c}\text { Revista de } \\
\text { Psiquiatria } \\
\text { Clínica | v. } 38 \text { | } \\
\text { n. } 2 \text { | } 2011 .\end{array}$ & Relato de caso único. & $\begin{array}{l}\text { Descrever a resposta de } \\
\text { um paciente com o tipo } \\
\text { generalizado de fobia } \\
\text { social e hiperidrose à Ter- } \\
\text { apia Cognitivo-Compor- } \\
\text { tamental aliada à toxina } \\
\text { botulínica. }\end{array}$ & A2 \\
\hline
\end{tabular}




\begin{tabular}{|c|c|c|c|c|}
\hline Nome do artigo & Publicação & Metodologia & Objetivo & WebQualis \\
\hline $\begin{array}{l}\text { Transtornos Fóbico-An- } \\
\text { siosos: Abordagem Epi- } \\
\text { demiológica das Inter- } \\
\text { nações Hospitalares }\end{array}$ & $\begin{array}{l}\text { Revista Cog- } \\
\text { itare Enfer- } \\
\text { magem | v. } 18 \\
\text { | n. } 1 \text { | p. 136- } \\
141 \text { | } 2013 .\end{array}$ & $\begin{array}{l}\text { Estudo descritivo e } \\
\text { retrospectivo realizado } \\
\text { no banco de dados do } \\
\text { Sistema de Internações } \\
\text { Hospitalares e Pron- } \\
\text { tuários, dos anos de } \\
1980 \text { a } 2005 .\end{array}$ & $\begin{array}{c}\text { Caracterizar os pacientes } \\
\text { hospitalizados acome- } \\
\text { tidos por transtornos } \\
\text { fóbico-ansiosos. }\end{array}$ & B2 \\
\hline $\begin{array}{c}\text { Transtornos Psiquiátricos } \\
\text { no Pós-Parto }\end{array}$ & $\begin{array}{c}\text { Revista de } \\
\text { Psiquiatria } \\
\text { Clínica | v. } 37 \text { | } \\
\text { n. } 6 \text { | } 2010 .\end{array}$ & $\begin{array}{l}\text { Revisão bibliográfica } \\
\text { acerca de transtornos } \\
\text { psiquiátricos no pós-par- } \\
\text { to a partir de artigos } \\
\text { encontrados no PubMed } \\
\text { e no SciELO entre os } \\
\text { anos de } 2000 \text { e } 2009 \text {. }\end{array}$ & $\begin{array}{c}\text { Avaliar transtornos } \\
\text { psiquiátricos no pós-par- } \\
\text { to. }\end{array}$ & B1 \\
\hline $\begin{array}{c}\text { Treinamento Físico Inter- } \\
\text { valado como Ferramenta } \\
\text { na Terapia Cogniti- } \\
\text { vo-Comportamental do } \\
\text { Transtorno de Pânico }\end{array}$ & $\begin{array}{l}\text { Jornal Brasilei- } \\
\text { ro de Psiqui- } \\
\text { atria | v. } 60 \text { | n. } \\
\qquad 3 \text { | } 2011 .\end{array}$ & Relato de experiência. & $\begin{array}{l}\text { Relatar a utilização de } \\
\text { um programa de exercíci- } \\
\text { os físicos aeróbicos inter- } \\
\text { valados no contexto da } \\
\text { Terapia Cognitivo-Com- } \\
\text { portamental em um caso } \\
\text { de transtorno de pânico } \\
\text { e agorafobia. }\end{array}$ & B1 \\
\hline $\begin{array}{l}\text { Tripofobia: Um Relato de } \\
\text { Caso do Tratamento do } \\
\text { Medo de Buracos }\end{array}$ & $\begin{array}{l}\text { Rev. Bras. de } \\
\text { Ter. Comp. } \\
\text { Cogn. | v. } 18 \text { | } \\
\text { n. } 2 \text { | p. } 100- \\
111 \text { | } 2016 .\end{array}$ & $\begin{array}{l}\text { Estudo baseado na } \\
\text { abordagem compor- } \\
\text { tamental, psicoeduca- } \\
\text { cional e de acompanha- } \\
\text { mento. }\end{array}$ & $\begin{array}{l}\text { Apresentar o caso de } \\
\text { uma jovem que se queix- } \\
\text { ava experimentar fortes } \\
\text { respostas fisiológicas, } \\
\text { como náusea e batimen- } \\
\text { to cardíaco acelerado, na } \\
\text { presença de estímulos. }\end{array}$ & B1 \\
\hline
\end{tabular}

\section{Resultados}

De acordo com a revisão integrativa realizada, percebe-se que, para o estudo da ansiedade no país, os artigos mostram que as possibilidades estratégicas perante esse transtorno se dão por duas categorias: o uso da Terapia Cognitivo-Comportamental (TCC), relatando as formas de tratamento para o TAG, a reestruturação cognitiva, exposição às situações temidas, treino de habilidades sociais e relaxamento; e o Tratamento Humanizado e Apoio Familiar, resultando, assim, em uma reintegração social maior e na melhora consequente nos sintomas apresentados pelo transtorno - sendo esta última categoria mais eficaz quando associada ao uso de outros tratamentos para a ansiedade.

\section{Discussão}

\section{Formas de Tratamento para o TAS}

Os autores que defendem a categoria de tratamento da TCC definem-na como uma associação a um bom funcionamento e resultados obtidos perante o transtorno de ansiedade. 
Dittz, Stephan, Gomes, Badaró e Lourenço (2015) descrevem o desenvolvimento de uma intervenção em grupo para pessoas que apresentaram os sintomas de TAS, fundamentada pela Terapia Cognitivo-Comportamental, utilizando técnicas como reestruturação cognitiva, enfrentamento às situações temidas, treino de habilidades sociais e relaxamento, bem como a abordagem da temática Depressão; já os autores Duailibi, Silva e Jubara (2013) falam sobre o transtorno depressivo que incapacita muitas pessoas com diversos sintomas que, quando não tratados, podem levar a muitas outras doenças, como infartos, Alzheimer, diabetes e acidente vascular isquêmico, assim como o uso de tratamentos por antidepressivos, os quais acabam com sintomas residuais que poderiam ser evitados pela utilização de outras formas de tratamento ou até mesmo a utilização associada deles.

Também, Chagas et al. (2010) falam sobre a busca pelo desenvolvimento de consensos de diagnósticos e tratamento para as doenças mais comuns diante do transtorno de ansiedade, tendo como resultados as manifestações clínicas, prejuízos e implicações, diferenças entre os subtipos generalizados e circunscritos, e o impacto com a depressão, abuso e dependência de drogas e outros transtornos de ansiedade. Levitan et al. (2011) discorrem sobre o desenvolvimento de consensos de diagnóstico e tratamento para as doenças mais comuns, tendo como resultados o tratamento farmacológico de primeira linha para adultos e crianças, inibidores seletivos de recaptação de serotonina e inibidores de recaptação de serotonina e noradrenalina, enquanto a Terapia Cognitivo-Comportamental é apontada como melhor tratamento psicoterápico.

Nesse sentido, as autoras Pezzato, Brandão e Oshiro (2012) relatam que as estratégias terapêuticas descritas como eficazes em transtorno de ansiedade envolvem procedimentos comportamentais e cognitivo-comportamentais de exposição ao enfrentamento de situações aversivas, tendo resultados para a efetividade dos procedimentos adotados, e confirmam a possibilidade de utilização da Psicoterapia Analítica Funcional (FAP) como eficaz ao tratamento do TA.

Os estudos realizados por King, Valença, Simões, Nardi e Silva (2012) acreditam que, com a verificação dos pacientes com transtorno de pânico e o uso da TCC, há melhoras em seus quadros quanto à ansiedade detectada e aos sintomas, em que cerca de $77 \%$ conseguem reduzir os ataques relacionados à ansiedade e melhoram os resultados de 55,8\% para 70,9\%. Com os estudos de Lessa e Fontenelle (2011), acredita-se que, com o uso da TCC, os pacientes conseguem ter uma melhora quanto aos sintomas apresentados em seus transtornos sociais. Assim, comprova-se a melhora dos sintomas dos transtornos, bem como os fenômenos periféricos ocorridos. Já Sardinha, Araújo e Nardi (2011) acreditam que, com o uso dos exercícios físicos e da TCC, há melhoras nos sintomas do transtorno de pânico e nos quadros ansiosos dos pacientes. E, ainda com relação ao transtorno de ansiedade apresentado em pacientes com tripofobia, os estudos de Marcone Reolon (2016) apontam que, com o uso da TCC, há melhoras consequentes nos sintomas apresentados pelo paciente, bem como sua reintegração social.

\section{Tratamento Humanizado e o Apoio Familiar}

Além desta categoria, o uso do tratamento mais humanizado, segundo as autoras Sousa, Verdana e Miasso (2016), fala sobre os fatores relacionados à adesão do tratamento 
medicamentoso por pessoas com transtorno de ansiedade, visando a uma melhora na qualidade de vida e possíveis benefícios de seu uso para a pessoa com ansiedade. O trabalho dos autores Bergerot, Laros e Araujo (2014) compara as vantagens e desvantagens psicométricas de instrumentos comumente utilizados em serviços especializados em Oncologia: Escala de Ansiedade e Depressão (HADS), Transtorno Geral de Ansiedade (GAD-7) e Questionário sobre Saúde do Paciente (PHQ-9). Eles defendem que esses pacientes, por possuírem uma doença como o câncer, estão mais vulneráveis e necessitam de um acompanhamento mais humanizado sobre seu psicológico e social.

O artigo das autoras Martinez et al. (2012) fala sobre os estudos quanto ao transtorno de ansiedade social, avaliando trabalhos sobre seu tratamento; comorbidade; validação/ propriedades psicrométricas; diagnóstico/prevalência; revisão crítica e teórica. Nota-se a importância de mais estudos sobre esse tema, para fornecer uma melhor compreensão do seu desenvolvimento e maior divulgação entre os profissionais de saúde para tornar o atendimento mais humanizado.

Santos, Goulart Júnior, Canêo, Lunardelli e Carvalho (2010) fizeram um levantamento bibliográfico sobre a neurose profissional, cujos sintomas são entendidos como a expressão simbólica de um conflito psíquico que se desenvolve a partir de uma situação organizacional ou profissional determinada.

Zanello e Silva (2012) acreditam que o transtorno de ansiedade e a depressão estão atingindo cada vez mais pessoas no mundo, assim, afirmam que a maior parcela da população que sofre com tais problemas é a feminina, composta por negras, pobres e domésticas, usuárias dos serviços de saúde pública, sendo elas medicalizadas de maneira exorbitante e com a necessidade de um tratamento mais adequado e humanizado. E, com a análise das alterações psicológicas sofridas no pós-parto e sua relação com o transtorno de ansiedade, Cantilino, Zambaldi, Sougey e Rennó (2010) acreditam que, com um tratamento mais humanizado e um maior acompanhamento clínico, há melhoras em seus quadros.

Nesse sentido, os autores Almeida, Silva, Espínola, Azevedo e Ferreira (2013) apresentam que o apoio familiar, na análise dos medos enfrentados pelos pacientes com o transtorno fóbico ansioso, é uma das formas de tratamento pontual utilizadas pelas pessoas com a doença. Paz, Brant, Marques e Machado (2013) afirmam que, com os pacientes fóbicos-ansiosos, é significativo o uso do tratamento precoce como uma contribuição para a melhora dos quadros apresentados em relação ao contexto social.

\section{Conclusão}

Evidencia-se que o TAG é uma patologia que vem se expandido nos últimos tempos, causando grande sofrimento à população. Pontuamos que esse transtorno é uma doença bem significativa, que acarreta um sofrimento físico e psíquico considerável e, como toda patologia, precisa ser compreendida e tratada em sua totalidade e de forma singular a cada indivíduo que a sofre.

Salientamos que uma das formas eficazes de tratamento é a preparação adequada da equipe. Ressalta-se, ainda, a importância da participação ativa da família desse doente no processo de prevenção e tratamento desse agravo. Assim, percebemos, com este estudo, que o uso da TCC é comprovadamente uma das formas de tratamento com melhoras no 
quadro dos pacientes analisados, da mesma forma que a utilização de métodos de abordagem mais humanizada e a interação social entre os pacientes e seus familiares, com o apoio e o uso associado com outras formas de tratamento, proporciona a consequente eficácia em diversas patologias ou quadros associados ao transtorno de ansiedade apresentados na maioria dos casos estudados.

Perante a importância da temática, ressaltamos a necessidade de realização de mais pesquisas que possam embasar não apenas teoricamente como também empiricamente os métodos efetivos que proporcionem meios de prevenção e tratamento a toda a população.

\section{Referências}

Almeida, P. A., Silva, P. M. C., Espínola, L. L., Azevedo, E. B., \& Ferreira, M. O., Filha (2013). Desafiando medos: relatos de enfrentamento de usuários com transtornos fóbicoansiosos. Revista Brasileira de Enfermagem, 66(4), 528-534. Disponível em http://www. scielo.br/pdf/reben/v66n4/v66n4a10.pdf

Andrade, L. H. S., Viana, M. C., \& Silveira, C. M. (2006). Epidemiologia dos transtornos psiquiátricos na mulher. Archives of Clinical Psychiatry, 33(2), 43-54. Disponível em https://www.revistas.usp.br/acp/article/view/17065/19060

Bergerot, C. D., Laros, J. A., \& de Araujo, T. C. C. F. (2014). Avaliação de ansiedade e depressão em pacientes oncológicos: Comparação psicométrica. Psico-USF, 19(2), 187-197. Disponível em http://www.scielo.br/pdf/pusf/v19n2/a02v19n2.pdf

Canguilhem, G. (2009). O normal e o patológico (6a ed.). Rio de Janeiro: Forense Universitária.

Cantilino, A., Zambaldi, C. F., Sougey, E. B., \& Rennó, J. O. E. L., Jr. (2010). Transtornos psiquiátricos no pós-parto. Archives of Clinical Psychiatry, 37(6), 288-294. Disponível em http://www.scielo.br/pdf/rpc/v37n6/a06v37n6

Castillo, A. R. G. L., Recondo, R., Asbahr, F. R., \& Manfro, G. G. (2000). Transtornos de ansiedade. Revista Brasileira de Psiquiatria, 22(Supl.2), 20-23. Disponível em http://www. scielo.br/pdf/rbp/v22s2/3791.pdf

Chagas, M. H. N., Nardi, A. E., Manfro, G. G., Hetem, L. A., Andrada, N. C. D., Levitan, M. N., . . \& Crippa, J. A. D. S. (2010). Diretrizes da Associação Médica Brasileira para o diagnóstico e diagnóstico diferencial do transtorno de ansiedade social. Revista Brasileira de Psiquiatria, 32(4), 444-452. Disponível em http://www.scielo.br/pdf/rbp/v32n4/a19v32n4.pdf

Craske, M., Stein, M. B., \& Hermann, R. (2014). Psychotherapy for generalized anxiety disorder. Disponível em www.uptodate.com

Dalgalarrondo, P. (2000). Psicopatologia e semiologia dos transtornos mentais. Porto Alegre: Artmed.

Dittz, C. P., Stephan, F., Gomes, D. A. G., Badaró, A. C., \& Lourenço, L. M. (2015). A terapia cognitivo-comportamental em grupo no Transtorno de Ansiedade Social. Estudos e Pesquisas em Psicologia, 15(3), 1061-1080. Disponível em https://www.e-publicacoes. uerj.br/index.php/revispsi/article/view/19428/14020

Dualibi, K., Silva, A. S. M., \& Jubara, C. F. B. (2013). Como diagnosticar e tratar a depressão. Revista Brasileira de Medicina, 71(12), 23-31.

King, A. L. S., Valença, A. M., Simões, J. P., Neto, Nardi, A. E., \& Silva, A. C. D. O. (2012). Subtipo respiratório versus não respiratório no transtorno de pânico com agorafobia: 
Avaliação com terapia cognitivo-comportamental. Psicologia: Reflexão e Crítica, 25(1), 41-47. Disponível em http://www.scielo.br/pdf/prc/v25n1/a06v25n1

Kinrys, G., \& Wygant, L. E. (2005). Transtornos de ansiedade em mulheres: Gênero influência o tratamento? Revista Brasileira de Psiquiatria, 27(2), 43-50. Disponível em http://www. scielo.br/pdf/rbp/v27s2/pt_a03v27s2.pdf

Lessa, L. R., \& Fontenelle, L. F. (2011). Toxina botulínica como tratamento para fobia social generalizada com hiperidrose. Revista de Psiquiatria Clínica, 38(2), 84-86. Disponível em https://www.revistas.usp.br/acp/article/view/17371/19408

Levitan, M. N., Chagas, M. H. N., Crippa, J. A. D. S., Manfro, G. G., Hetem, L. A., Andrada, N. C., . . \& Nardi, A. E. (2011). Diretrizes da Associação Médica Brasileira para o tratamento do transtorno de ansiedade social. Revista Brasileira de Psiquiatria, 33(3), 292-302. Disponível em http://www.scielo.br/pdf/rbp/v33n3/14.pdf

Marcon, R. M., \& Reolon, G. A. (2016). Tripofobia: Um relato de caso do tratamento do medo de buracos. Revista Brasileira de Terapia Comportamental e Cognitiva, 18(2), 100-111. Disponível em http://www.usp.br/rbtcc/index.php/RBTCC/article/view/886/482

Martinez, A. S., Oliveira, A. M., Badaró, A. C., Gomes, D. A. G., Peres, F. S., Tavares, F. S., . . \& \& Lourenço, L. M. (2012). Periódicos da CAPES: perspectiva das dissertações e teses sobre transtorno de ansiedade social/fobia social. Interação em Psicologia, 16(2). Disponível em https://revistas.ufpr.br/psicologia/article/view/24730/20283

Moher, D., Liberati, A., Tetzlaff, J., Altman, D. G., \& PRISMA Group (2009). Preferred reporting items for systematic reviews and meta-analyses: The PRISMA statement. Annals of Internal Medicine, 151(4), 264-269. doi: https://doi.org/10.1371/journal.pmed.1000097

Nardi, A. E. (2006). Some notes on a historical perspective of panic disorder. Jornal Brasileiro de Psiquiatria, 55(2), 154-160. Disponível em http://www.scielo.br/scielo. php?pid=S0047-20852006000200010\&script=sci_arttext

Organização Mundial da Saúde. (2016). Saúde mental: Nova concepção, nova esperança. Genebra: Organização Mundial da Saúde. Disponível em http://www.who.int/whr/2001/ en/whr01_po.pdf

Paz, C. C. P., Brant, I. L. M., Marques, M. S., \& Machado, R. M. (2013). Transtornos fóbico-ansiosos: Abordagem epidemiológica das internações hospitalares. Cogitare Enfermagem, 18(1), 136-141. Disponível em https://www.scielosp.org/scielo. php?pid=S1413-81232016000200591\&script=sci_arttext\&tlng=en

Pezzato, F. A., Brandão, A. S., \& Oshiro, C. K. B. (2012). Intervenção baseada na psicoterapia analítica funcional em um caso de transtorno de pânico com agorafobia. Revista Brasileira de Terapia Comportamental e Cognitiva, 14(1), 74-84. Disponível em http://pepsic. bvsalud.org/pdf/rbtcc/v14n1/v14n1a06.pdf

Sampaio, R. F., \& Mancini, M. C. (2007). Estudos de revisão sistemática: Um guia para síntese criteriosa da evidência científica. Revista Brasileira de Fisioterapia, 11(1), 83-89. Disponível em http://www.scielo.br/pdf/rbfis/v11n1/12.pdf

Santos, L. C., Goulart Júnior, E., Canêo, L. C., Lunardelli, M. C. F., \& Carvalho, P. L. T. D. (2010). Psicologia e profissão: Neurose profissional e a atuação do psicólogo organizacional frente à questão. Psicologia: Ciência e Profissão, 30(2), 248-261. Disponível em http:// www.scielo.br/pdf/pcp/v30n2/v30n2a03 
Sardinha, A., Araújo, C. G. S., \& Nardi, A. E. (2011). Treinamento físico intervalado como ferramenta na terapia cognitivo-comportamental do transtorno de pânico. Jornal Brasileiro de Psiquiatria, 60(3), 227-230. Disponível em http://psicologalaismutuberria. com/admin/data/uploads/artigos-pdf/89943f483511f31b69a837c9fd86c4c0.pdf

Sousa, L. P. C., Verdana, K. G. G., \& Miasso, A. I. (2016). Adesão ao tratamento medicamentoso por pessoas com transtorno de ansiedade. Cogitare Enfermagem, 21(1), 1-11. Disponível em https://revistas.ufpr.br/cogitare/article/view/43510/27515

Zanello, V., \& Silva, R. M. C. (2012). Saúde mental, gênero e violência estrutural. Revista Bioética, 20(2), 267-79. Disponível em http://www.revistabioetica.cfm.org.br/index.php/ revista_bioetica/article/view/745/776

Zuardi, A. W. (2017). Características básicas do transtorno de ansiedade generalizada. Medicina (Ribeirão Preto, On-line.), 50(Supl.1), 51-55. Disponível em http://www. periodicos.usp.br/rmrp/article/view/127538/124632

Recebido em: 24/02/2019

Última revisão: 07/03/2019

Aceite final: 07/06/2019

\section{Sobre os autores:}

Lívia Ivo D’ávila - Psicóloga. Faculdade de Saúde Ibituruna (FASI). E-mail: liviaidavila1@gmail.com, Orcid: http://orcid.org/0000-0002-4823-1380

Fernanda Cardoso Rocha - Mestranda em Cuidados Primários em Saúde pela Universidade Estadual de Montes Claros (UNIMONTES). Especialista em Saúde da Família. Especialista em Metodologia e Didática do Ensino Superior. MBA em Gestão de Recursos Humanos. Especialista em Psicologia Hospitalar. Psicóloga. E-mail: nandac.rocha@hotmail.com, Orcid: http://orcid.org/0000-0001-6381-2063

Bruna Roberta Meira Rios - Especialista em Enfermagem Cardiológica. Enfermeira. Docente das Faculdades de Saúde Ibituruna (FASI). E-mail: brunameirarios@gmail.com, Orcid: http://orcid.org/0000-0002-7715-2921

Sabrina Gonçalves Silva Pereira - Mestre em Ciências da Saúde pela Universidade Estadual de Montes Claros (UNIMONTES). Enfermeira. Docente das Faculdades de Saúde Ibituruna (FASI). E-mail: sabrina.goncalves@fasi.edu.br, Orcid: http://orcid.org/0000-0001-8737-6569

Álvaro Parrela Piris - Mestre em Tecnologia Aplicada à Saúde pelas Faculdades Promove-BH. Docente das Faculdades de Saúde Ibituruna (FASI). E-mail: alvaroparrela@fasi.edu.b, Orcid: http://orcid.org/0000-0002-7151-5015 